\title{
Correction: VersicanV1 promotes proliferation and metastasis of hepatocellular carcinoma through the activation of EGFR-PI3K-AKT pathway
}

Guangyan Zhangyuan $^{1,2} \cdot$ Fei Wang ${ }^{1,2,3} \cdot$ Haitian Zhang ${ }^{1,2,3} \cdot$ Runqiu Jiang $^{1,2,3} \cdot$ Xuewen Tao $^{1,2} \cdot$ Decai Yu $^{2}$. Kangpeng $\mathrm{Jin}^{2,3} \cdot$ WeiWei $\mathrm{Yu}^{2} \cdot$ Yang $\mathrm{Liu}^{2,3} \cdot \mathrm{Yin} \mathrm{Yin}^{2} \cdot$ Jintao Shen ${ }^{4} \cdot$ Qinfeng $\mathrm{Xu}^{4} \cdot$ Wenjie Zhang ${ }^{1,2,3}$. Beicheng Sun ${ }^{1,2,3}$

Published online: 7 November 2019

(c) The Author(s), under exclusive licence to Springer Nature Limited 2019

\section{Correction to: Oncogene}

https://doi.org/10.1038/s41388-019-1052-7
The original version of this article did not acknowledge Beicheng Sun as a corresponding author. This has now been corrected in both the PDF and HTML versions of the article.

Wenjie Zhang

drzhangwj@163.com

$\triangle$ Beicheng Sun

sunbc@nju.edu.cn

1 Department of Hepatobiliary Surgery of Drum Tower Clinical Medical College, Nanjing Medical University, Nanjing, PR China

2 Department of Hepatobiliary Surgery, The Affiliated Drum Tower Hospital of Nanjing University Medical School, Nanjing, Jiangsu, PR China

3 Liver Transplantation Center of the First Affiliated Hospital and State Key Laboratory of Reproductive Medicine, Nanjing Medical University, Nanjing, Jiangsu, PR China

4 Department of Nuclear Medicine, The Affiliated Drum Tower Hospital of Nanjing University Medical School, Nanjing, Jiangsu, PR China 